\title{
THE SINGLETON SET TOPOLOGICAL PROPERTY; TINY BUT POWERFUL
}

\section{CHARLES DORSETT}

Department of Mathematics

Texas A\&M University Commerce

Commerce, Texas 75429

USA

e-mail: charles.dorsett@tamuc.edu

\begin{abstract}
Within this paper, the known strong properties of the singleton set topological property are expanded to include separation axioms and those properties are used to characterize the singleton set topological property.
\end{abstract}

\section{Introduction and Preliminaries}

By 1890 the study of mathematics was flourishing, but there was a problem; there was no common language or classification used in the study of mathematics and communication amongst the different groups working on mathematics was inhibited. To overcome that difficulty, in 1892, Riemann undertook the task of classifying spaces for use by all. Each of Frechet, Riesz, and Weyl made substantial contributions to the task undertaken by Riemann and the classification culminated in 1914 with the epic paper by Hausdorff, who found the right axiomatic system 2010 Mathematics Subject Classification: 54B05, 54B10, 54C05, 54C10.

Keywords and phrases: image properties, subspace properties, product properties, the singleton set topological property.

Received May 22, 2019

(C) 2019 Scientific Advances Publishers 
for Weyl's neighbourhoods, making them a suitable abstraction and giving birth of modern topology [16]. Those mathematical ancestors and others did an incredible job giving mathematicians a common language and classification to more easily communicate with each other and more quickly extend the frontiers of mathematics. Below all spaces are topological spaces.

To add structure to spaces and make spaces more applicable, properties were added. Of concern and importance were properties preserved by homeomorphisms; one-to-one, onto, continuous, and open functions, which are called topological properties. Given a property for a space, there are many questions to be asked and resolved, including the following; (1) Is the property a topological property?, (2) If $P$ is not a topological property, what can be said about each of continuous, continuous open, open, continuous closed, and closed images of a space with property $P$ ?, (3) Given two properties $P$ and $Q$ of a space, are the two properties topologically related or are they independent of each other?, (4) Is a topological property $P$ a subspace property?, and (5) Is a property $P$ of a space a product property?

In classical topology, a property $P$ was labelled a continuous image property if for each space $(X, T)$ with property $P$, each continuous image of $(X, T)$ has property $P$. In a similar manner, each of continuous open, open, continuous closed, and closed image properties were defined. However, in the many classical studies of topological properties, because of the many, very different known topological properties, and no recognized topological tool to address the great variety of topological properties, the existence of a least topological property was never considered, which, if such a topological property exists, could create problems. 
As is often the case, progress was made on the resolution of the question of a least topological property in the further investigation of $T_{0}$-identification spaces, which were introduced in 1936 [12].

Definition 1.1. Let $(X, T)$ be a space, $R$ be the equivalence relation on $X$ defined by $x R y$ iff $C l(\{x\})=C l(\{y\})$, let $X_{0}$ be the set of $R$ equivalence classes on $X$, let $N:(X, T) \rightarrow\left(X_{0}, Q(X, T)\right)$ be the natural map, and let $Q(X, T)$ be the decomposition topology on $X_{0}$ determined by $(X, T)$ and the map $N$. Then $\left(X_{0}, Q(X, T)\right)$ is the $T_{0}$-identification space of $(X, T)$.

In the 1936 paper [12], $T_{0}$-identification spaces were shown to be $T_{0}$ and used to jointly characterize pseudometrizable and metrizable.

Theorem 1.1. Let $(X, T)$ be a space. Then $(X, T)$ is pseudometrizable iff $\left(X_{0}, Q(X, T)\right)$ is metrizable.

Thus for a topological property $P$ for which $P o=\left(P\right.$ and $\left.T_{0}\right)$ exists, the question of what topological property $Q$, if any, would a space $(X, T)$ have property $Q$ iff $\left(X_{0}, Q(X, T)\right)$ has property $P o$ arose, leading to the introduction and investigation of weakly Po properties [2].

Definition 1.2. Let $P$ be a topological property for which $P o=\left(P\right.$ and $\left.T_{0}\right)$ exists. Then a space is weakly $P o$ iff its $T_{0}$-identification space has property $P o$. A topological property $P o$ for which weakly $P o$ exists is called a weakly $P o$ property.

In the introductory weakly $P o$ property paper [2], the search for a topological property that is not a weakly Po property led to a need and use for the topological property "not- $T_{0}$ ", where "not- $T_{0}$ " is the negation of $T_{0}$. Thus "not- $T_{0}$ " proved to be a needed, useful topological property, which led to the addition of the topological properties "not- $P$ ", where $P$ is 
topological property for which "not- $P$ " exists, as important, useful topological properties in the study of topology. The upgrade of "not- $P$ " to a topological property and the realization that "not- $P$ ", where $P$ is a topological property for which "not- $P$ " exists, could be an important, useful topological tool led to a quick, easily understandable resolution to the question above concerning a least topological property.

Theorem 1.2. $L=\left(T_{0}\right.$ or "not $-T_{0}$ " $)=(P$ or "not-P"), where $P$ is a topological property for which "not-P" exists, is the least of all topological properties [3].

Thus "not- $P$ ", where $P$ is a topological property for which "not- $P$ " exists, was precisely the right tool needed to resolve the difficult, long unaddressed question above concerning a least topological property. Also, "not- $P$ ", where $P$ is a topological property for which "not- $P$ " exists, proved to be the tool that could be quickly and easily used to prove there is no strongest topological property [4]. Thus, the use of "not- $P$ " as a topological property quickly and easily resolved another difficult, long unaddressed question in the study of topology.

In the 2016 paper [5], it was shown that every space has property $L$, which was used in the paper [6] to establish $L$ is a continuous image property using the classical definition of continuous image properties. Thus every space exhibits the continuous image property $L$, which is far from the intent of the creators of continuous image properties, creating a disconnect in the study of continuous images properties. As a result, to maintain continuity between past studies of continuous image properties and future studies of continuous image properties, a correction in the definition of continuous image properties was needed. Since $L$ created the disconnect, then removal of $L$ as a continuous image property would end the disconnect. 
Definition 1.3. A topological property $P$ is a continuous image property iff $P \neq L$ and every continuous image of a space with property $P$ has property $P[6]$.

In the 2016 paper [6], the singleton set property was defined and shown to be the strongest topological property that is a continuous image property.

Definition 1.4. A space $(X, T)$ has the singleton set property iff $X$ is a singleton set.

Thus, the singleton set property is tiny, but powerful. Are there other topological properties with strongest topological property the singleton set property?.

In the 2016 paper [7], each of open and continuous open image properties were further investigated. As in the case of continuous image properties, $L$ created a disconnect in the study of each of open and continuous open image properties, which was corrected with the removal of $L$ as an open and a continuous open image property, and it was shown that the singleton set property is the strongest of both the open and continuous open image properties.

In the 2016 paper [8], each of closed and continuous closed image properties were further investigated and, as above, $L$ created a disconnect in the study of each of closed and continuous closed image properties, which was corrected with the removal of $L$ as a closed and a continuous closed image property, and it was shown that the singleton set property is the strongest of both the closed and continuous closed image properties.

Subspace properties have been long studied in mathematics. As above, the existence of $L$ created a disconnect in the study of subspace properties, which was corrected with the removal of $L$ as a subspace property and it was shown that the singleton set property is the strongest of the subspace properties [9]. 
Topological product properties were introduced in 1930 [14].

Definition 1.5. Let $P$ be a topological property. Then $P$ is a product property iff a product space, with the Tychonoff topology, has property $P$ iff each factor space has property $P$.

As above, the existence of $L$ created a disconnect in the study of product properties, which was ended with the removal of $L$ as a product property. Within the 2017 paper [9], it was shown that the singleton set property is the strongest of the product properties.

Hence, the singleton set property, as advertised above, is tiny, but powerful. Are there additional topological properties with strongest topological property the singleton set property? Below this question is addressed and the results both above and below are used to give characterizations of the singleton set property.

\section{The Singleton Set Property and Separation Axioms}

In Hausdorff's 1914 paper [10], he introduced a topological property that became known as the Hausdorff property or the $T_{2}$ property.

Definition 2.1. A space $(X, T)$ is Hausdorff, or equivalently $T_{2}$, iff for distinct elements $x$ and $y$ in $X$, there exist disjoint open sets $U$ and $V$ such that $x \in U$ and $y \in V$.

Thus, the Hausdorff property was the first of the topological properties that topological separated two distinct elements in a topological space. Others such properties include $T_{0}, T_{1}$, and Urysohn.

In the 1943 paper [11], $T_{1}$ was generalized to $R_{0}$.

Definition 2.2. A space $(X, T)$ is $R_{0}$ iff for each closed set $C$ and each $x \notin C, C \cap C l(\{x\})=\phi$.

In the 1961 paper [1], $T_{2}$ was generalized to $R_{1}$. 
Definition 2.3. A space $(X, T)$ is $R_{1}$ iff for $x$ and $y$ in $X$ such that $C l(\{x\}) \neq C l(\{y\})$, there exist disjoint open sets $U$ and $V$ such that $x \in U$ and $y \in V$.

In 1921, Vietoris [15] introduced a topological property that topologically separates a closed set and an element not in the closed set, called the regular property.

Definition 2.4. A space $(X, T)$ is regular iff for each closed set $C$ and each $x \notin C$, there exist disjoint open set $U$ and $V$ such that $x \in U$ and $C \subseteq V$. A regular $T_{1}$ space is denoted by $T_{3}$.

There are other classical topological properties that topological separates closed sets and elements not in the closed set.

In 1923, Tietze [13] introduced the normal topological property.

Definition 2.5. A space $(X, T)$ is normal iff for disjoint closed sets $C$ and $D$, there exist disjoint open sets $U$ and $V$ such that $C \subseteq U$ and $D \subseteq V$. A normal $T_{1}$ space is denoted by $T_{4}$.

There are other classical topological properties that topologically separate disjoint closed sets.

In the 1923 paper [13], Tietze collectively labelled all those topological properties that topological separates two elements or closed sets and an element not in the closed set or two disjoint closed sets as separation axioms. Thus, in a trivial manner, the singleton set property is the strongest of the separation axioms.

For the properties given in the section above, using their classical definitions, $L$ satisfied the properties. Is $L$ a separation axiom? The following example shows the answer to the question is no. 
Example 2.1. Let $X=\{a, b, c, d\}$ and $T=\{\{a, b\},\{c\},\{a, b, c\},\{c, d\}\}$. Then $(X, T)$ has property $L$, but satisfies none of the separation axioms.

Below, the above results are used to characterize the singleton set property.

\section{Characterizations of the Singleton set Property}

Theorem 3.1. Let $(X, T)$ be a space. Then the following are equivalent: (a) $(X, T)$ has the singleton set property, (b) $(X, T)$ simultaneously satisfies every continuous image property, (c) $(X, T)$ simultaneously satisfies every continuous open image property, $(\mathrm{d})(X, T)$ simultaneously satisfies every continuous closed image property, (e) $(X, T)$ simultaneously satisfies every open image property, (f) $(X, T)$ simultaneously satisfied every closed image property, $(\mathrm{g})(X, T)$ simultaneously satisfies every subspace property, (h) $(X, T)$ simultaneously satisfies every product property, and (i) $(X, T)$ simultaneously satisfies every separation axiom.

Proof. (a) implies (b): Since the singleton set property is the strongest of the continuous image properties, then (a) implies (b).

(b) implies (a): Since the singleton set property is a continuous image property, then (b) implies (a).

In a similar manner the remainder of the theorem can be proven.

Thus the singleton set property, which in the past was used to give a quick, easily seen example of a topological space, has a much bigger role in the study of topology and is precisely the topological tool needed to resolve past, unaddressed questions concerning a strongest topological property. The singleton set property is tiny, but, without question, powerful. 


\section{References}

[1] A. Davis, Indexed systems of neighborhoods for general topological spaces, American Mathematical Monthly 68(9) (1961), 886-893.

DOI: http://dx.doi.org/10.2307/2311686

[2] C. Dorsett, Weakly $P$ properties, Fundamental Journal of Mathematics and Mathematical Sciences 3(1) (2015), 83-90.

[3] C. Dorsett, Weakly $P$ corrections and new, fundamental topological properties and facts, Fundamental Journal of Mathematics and Mathematical Sciences 5(1) (2016), 11-20.

[4] C. Dorsett, Another important use of "not- $P$ ", where $P$ is a topological property, Pioneer Journal of Mathematics and Mathematical Sciences 18(2) (2016), 97-99.

[5] C. Dorsett, Pluses and needed changes in topology resulting from additional properties, Far East Journal of Mathematical Sciences 101(4) (2016), 803-811.

DOI: http://dx.doi.org/10.17654/MS101040803

[6] C. Dorsett, Applications of new topological properties in the study of singleton set, indiscrete, and continuous image properties, Journal of Mathematical Sciences: Advances and Applications 39 (2016), 27-38.

DOI: http://dx.doi.org/10.18642/jmsaa_7100121659

[7] C. Dorsett, The singleton set property, and open and continuous open image properties, Fundamental Journal of Mathematics and Mathematical Sciences 5(2) (2016), 57-64.

[8] C. Dorsett, The singleton set property, and closed and continuous closed image properties, Pioneer Journal of Mathematics and Mathematical Sciences 8(1) (2016), 99-108.

[9] C. Dorsett, Intersection existent and least and strongest properties for topological properties, product properties, subspace properties, and properties that are weakly Po, Pioneer Journal of Mathematics and Mathematical Sciences 19(1) (2017), 37-44.

[10] F. Hausdorff, Grundzuge der Mengenlehre, Leipzig (2014); Reprinted by Chelsea, New York, 1949 [MR 11, p.88].

[11] N. Shanin, On separations in topological spaces, Akademiia Nauk SSSR Comptes Rendus (Doklady) 38 (1943), 110-113.

[12] M. H. Stone, Application of Boolean algebras to topology, Matematicheskii Sbornik 1(43) Number (5) (1936), 765-772. 
[13] H. Tietze, Beitrage zur allgemeinen topologie: I, Mathematische Annalen 88(3-4) (1923), 290-312.

DOI: https://doi.org/10.1007/BF01579182

[14] A. Tychonoff, Uber die Topologische Erweiterung von Raumen, Mathematische Annalen 102(1) (1930), 544-561.

DOI: https://doi.org/10.1007/BF01782364

[15] L. Vietoris, Stetige Mengen, Monatshefte für Mathematik 31(1) (1921), 173-204.

DOI: https://doi.org/10.1007/BF01702717

[16] S. Willard, General Topology, Addison-Wesley Publ. Co., 1970. 